\title{
Use of reciprocity considerations for the two-dimensional BEM analysis of wave propagation in an elastic half-space with applications to acoustic emission
}

\author{
Irene Arias*, Jan D. Achenbach \\ Center for Quality Engineering and Failure Prevention, Northwestern University, Evanston, IL 60208, USA
}

Received 16 September 2003; received in revised form 16 November 2003; accepted 20 November 2003

\begin{abstract}
A simple numerical treatment of the infinite boundary in the BEM analysis of two-dimensional wave propagation problems in elastic half-spaces is proposed to avoid the spurious reflections of non-decaying Rayleigh waves introduced by the truncation of the boundary. The proposed method exploits the knowledge of the far-field asymptotic behavior of the solution to adequately correct the BEM displacement system matrix for the truncated problem to account for the contribution of the omitted part of the boundary. The reciprocal theorem of elastodynamics is used for a convenient computation of this contribution exclusively in terms of the boundary integrals of the original BEM system. The method is applied to the study of the acoustic emission from nucleating and propagating surface-breaking and buried cracks in a two-dimensional elastic half-space. It is shown to be particularly advantageous since it allows for an accurate calculation of the generated signal even when the observation point is located far from the acoustic emission source.
\end{abstract}

(C) 2003 Elsevier B.V. All rights reserved.

\section{Introduction}

The boundary element method (BEM) is ideally suited for the numerical analysis of problems of wave scattering by flaws such as cracks, cavities, and inclusions in elastic media. It has been successfully applied to the solution of two- and three-dimensional problems in the frequency domain and for unbounded or partially bounded elastic bodies. The most important feature of this numerical method is that it only requires the discretization of the boundary rather than the entire domain. In addition, the radiation condition at infinity is naturally included in the formulation. Therefore, it is particularly well suited for unbounded domains. However, the analysis of wave propagation in two-dimensional elastic half-spaces presents some difficulties, since the boundary is infinite. In elastodynamics, the BEM formulation for a half-space is usually stated in terms of full-space-rather than half-space—Green's functions, and thus the discretization over the boundary of the half-space is needed in order to enforce the appropriate boundary conditions. Obviously, the infinite surface of the half-space has to be truncated for computational purposes. The simple truncation of the boundary produces spurious reflections of elastic waves at the limits of the computational

* Corresponding author. Present address: Graduate Aeronautical Laboratories, California Institute of Technology, 1200 E. California Blvd. MC: 205-45, Pasadena, CA 91125, USA. Tel.: +1-626-395-4757; fax: +1-626-449-2677.

E-mail addresses: iarias@caltech.edu (I. Arias), achenbach@ northwestern.edu (J.D. Achenbach). 
domain. This approach can lead to accurate results in the three-dimensional case, since all types of waves exhibit geometrical attenuation and thus their reflections are not significant provided the discretization is extended far enough. However, in the two-dimensional case, Rayleigh waves propagate along the surface of the half-space without attenuation. Consequently, the spurious reflections are generally significant, independently of the extent of he discretization. In the frequency donain approach, this issue is typically addressed by adding a small anount f damping to the material. More sophisticated appro aches include the infinite boundary element technique, fir proposed in [1]. This technique maps the omitted part of the boundary, which is is The behavior of the displacements and suitable for each particular problem. In volving Rayleigh waves [2,3]. In all ca tegration schemes and are particularly inv In previous work [4], we have propo a convenient computation of this contribution involving the same element integrals that form the original BEM system. The proposed method is easy to implement and, in the present case, it comes at essentially no additional cost as compared to the simple truncation of the boundary. The accuracy of the solution provided by the proposed model depends on the accuracy of the assumption that Rayleigh waves strongly dominate at the end points of the computational domain. However, once the computational domain is extended far enough from the source region for this assumption to hold, then, unlike the simply truncated solution, the corrected solution is accurate everywhere in the computational domain and for all computed times. In situations where the region of interest extends far beyond the source region where waves are generated, as is commonly the case in acoustic emission configurations, the proposed method reduces the extent of the computational domain.

In this paper, the quantitative characterization of $\mathrm{AE}$ events is explored computationally for a selected set of examples. The acoustic emission from nucleating surface-breaking and buried cracks is studied, and the generated surface motions in a two-dimensional homogeneous, isotropic, linearly elastic half-space are analyzed by the boundary element method in the frequency domain. The numerical approach considers defects of finite sizes, and computes the surface disturbances both in the near and far-fields. Only cracks normal to the surface are considered here. However, the presented computational approach can also be used to address more general configurations and geometries.

Acoustic emissions (AEs) are transient stress waves within solids radiated from localized sudden changes in the stress state. They are usually associated with damage events in the material, such as crack nucleation and growth, plastic activity, or various debonding and fracture mechanisms in composite materials. This wave motion propagates through the solid and eventually produces disturbances in the surface, which in principle can be detected. Thus, acoustic emission can be used to monitor the damage activity in specimens, provided that the generated signals are large enough relative to the noise level. Acoustic emission techniques have been used in various situations, from analyzing the development of texture in martensitic materials [5], to the monitoring of corrosion processes and welds in pressure vessels and bridges in service. Acoustic emission has also been applied to monitoring the fatigue crack growth in laboratory tests. A particularly fertile field of application is the analysis of damage in composite materials containing at least a brittle phase [6].

Acoustic emission techniques are quite different from other nondestructive evaluation (NDE) methods since internal flaws developing or evolving in all the sample can be detected by taking measurements in a limited region of the surface of the sample. Furthermore, AE techniques allow for continuous monitoring of components while they are in use, and damage events are active. This contrasts with conventional nondestructive techniques which analyze the integrity of components after all events have occurred. Nevertheless, AE as a nondestructive testing technique 
presents several problems. Due to reflections with boundaries and other features of the sample, the recorded signals depend on the overall structure. It is very difficult to establish a direct correspondence between a disturbance on the surface and the particular event that caused it. Moreover, the detection threshold is often hindered by the background noise, and weak AE events may remain unnoticed. This is particularly important since the AE signals cannot be

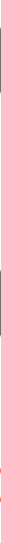

Theoretical efforts have been made to quantitatively analyze AE events. One approach borrowed from seismology consists in the representation of the AE process by point-sources, analogously to the shear dipole point representation in laser generation of ultrasound [13]. The force dipole tensor provides a simple and convenient way to represent various types of fracture events, while retaining the fundamental physics [7]. This approach however does not account for the finite extent of the defect, and is therefore valid only in the far-field. Besides, it assumes that all the stress changes in the fracture event occur simultaneously, i.e. it does not address the dynamic crack propagation. A more detailed asymptotic analysis including the finite size, the curvature of the crack front, and the crack propagation speed in an unbounded solid has been developed in [8]. In particular, this analysis showed that brittle events generate stronger acoustic emission signals than ductile crack propagation. Similar analysis of the stress-waves radiated from sudden activity at the crack tip in an infinite body has been reported in [14]. In [15] the disturbances generated by the fracture processes of a buried penny-shaped crack on the free surface of a half-space were analyzed.

The present paper is organized as follows. The proposed approach to model the acoustic emission from nucleating and propagating surface-breaking and buried cracks in an elastic solid is described in detail in Section 2. The elastic solid is modeled as a homogeneous, isotropic, linearly elastic half-space. Since only cracks normal to the surface are considered, symmetry arguments restrict the analysis to a quarter-space. In Section 3, the numerical technique for the treatment of the infinite surface of the quarter-space is formulated and its implementation in a frequency domain boundary element scheme is discussed. Selected numerical examples are presented in Section 4 to illustrate some features of the surface disturbances and the application of the presented computational method to a relevant field in NDE.

\section{Modeling approach}

We consider a homogeneous, isotropic, linearly elastic half-space subject to a uniform tensile stress at infinity $\sigma_{\infty}$ parallel to the surface (see Fig. 1). By virtue of linear superposition, the total field is decomposed into the incident and emitted fields. The uniform stress field can be understood as a static incident field. The nucleation of a crack is viewed as a sudden release of the corresponding traction on the crack faces. Consequently, it is analyzed by considering the field generated by the sudden application of a horizontal traction of $-\sigma_{\infty} H(t)$ on the crack faces, $H(t)$ being the Heaviside step function. Thus, similar to the point-source representation, we assume that all the changes in the source occur simultaneously. We consider surface-breaking cracks, as well as buried cracks. Crack growth is modeled by suddenly releasing the asymptotic crack tip field [16], as illustrated in Fig. 2. Again, it is clear 

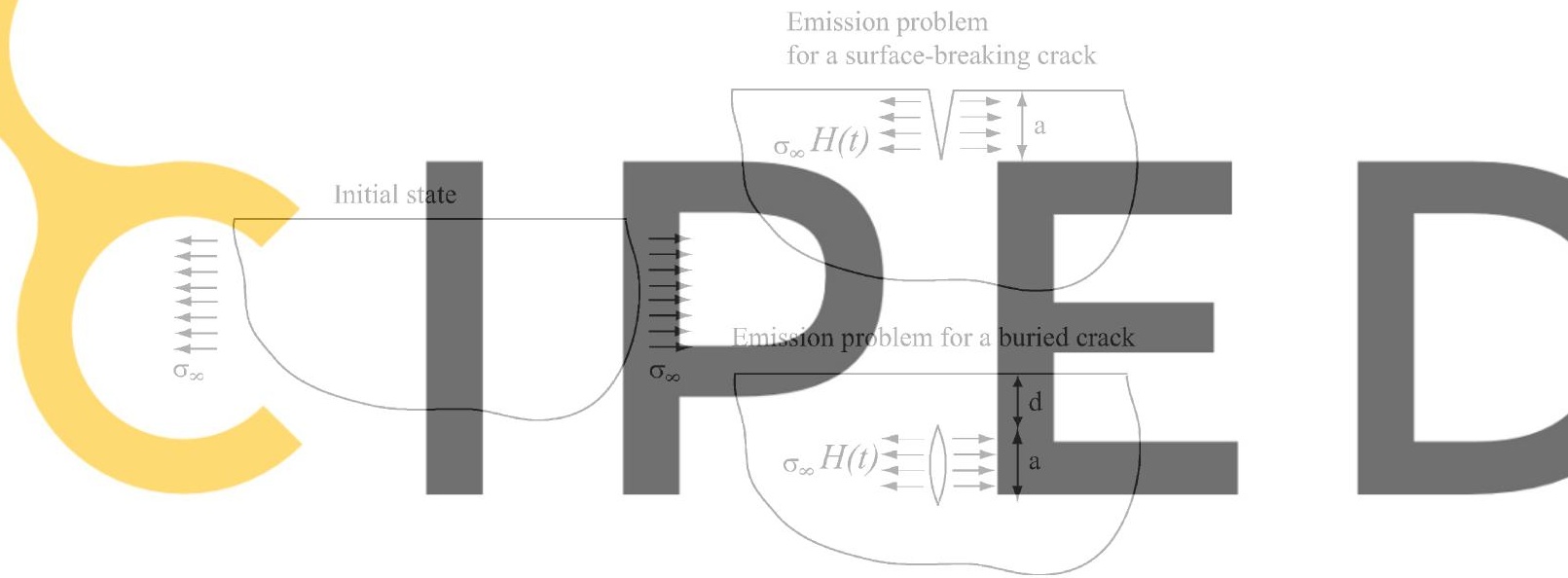

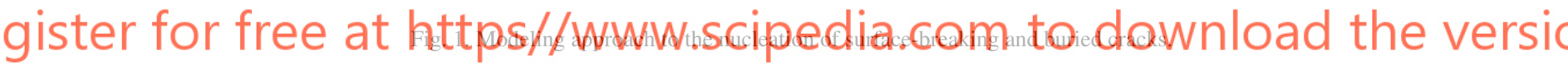

that this approach does not address the dynamic crack propagation phenomenon. Furthermore, the increment of the crack must be small compared to other dimensions of the problem for the asymptotic crack field solution to be valid. A singular traction quarter-point (STQP) element has been used to reproduce the $r^{-1 / 2}$ singularity of the asymptotic stress field at the crack tip. By displacing the mid-node of a quadratic boundary element with straight-line geometry to a quarter of its length and adequately modifying the element shape functions, the interpolated traction field in the element exhibits the appropriate asymptotic behavior at the crack tip [17].

The release of the stress in the crack faces is treated numerically by replacing the Heaviside step function by $S$-shaped functions $S_{\alpha}(t)$. The sharpness of this regularized step, which is controlled by the parameter $\alpha$, is a simple model for the brittleness or ductility of the fracture process. The considered $S$-shaped functions are of the form (Fig. 3)

$$
S_{\alpha}(t)=\frac{1}{1+\mathrm{e}^{\left(t-t_{0}\right) / \alpha}},
$$

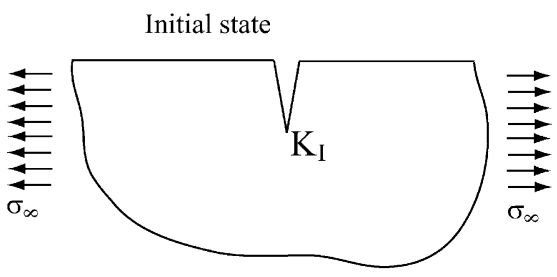

Emission problem
for a surface-breaking crack

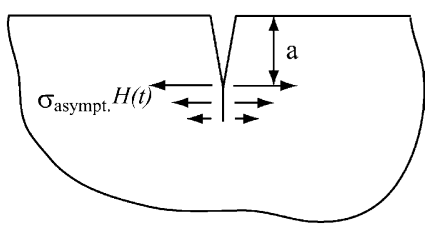

Emission problem for a buried crack
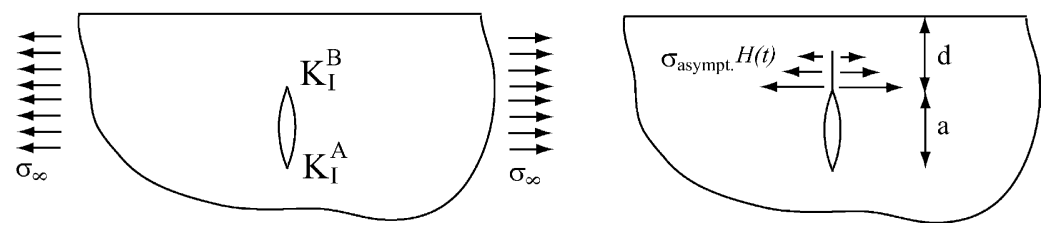

Fig. 2. Modeling approach to the propagation of surface-breaking and buried cracks. 

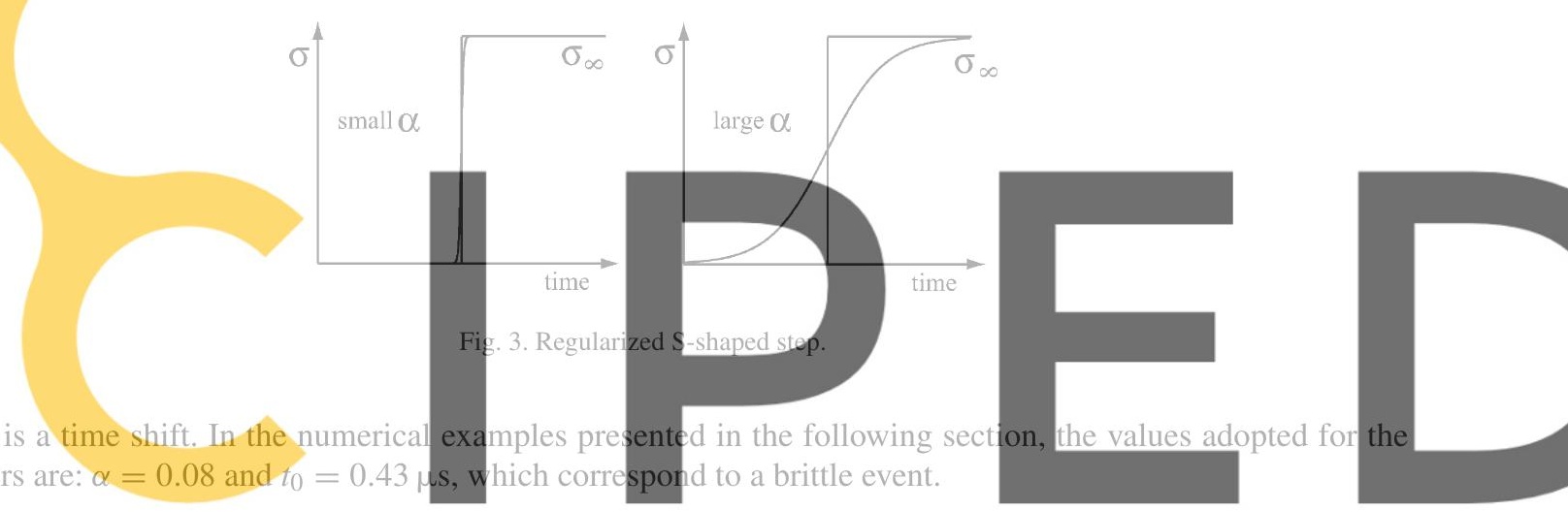

\section{gister for free at https//wWw.scipedia.com to download the versic}

The above described elastodynamic problems for a two-dimensional half-space are solved numerically by the direct frequency domain boundary element method with quadratic interpolations. The symmetry of the emission problems allows us to restrict the analysis to a quarter-space. In the following, we present a numerical technique which allows the undamped Rayleigh waves propagating along the infinite surface of the quarter-space to escape the computational domain without producing spurious reflections from its limits. It should be noted that the vertical boundary of the quarter-space is a fictitious boundary resulting from symmetry considerations. Therefore, no Rayleigh waves travel along this boundary and it can be truncated at a sufficiently large distance without loss of accuracy.

The frequency domain boundary integral equation for a point $\xi$ on the boundary of the quarter-space $\Gamma$ in the absence of body forces may be obtained from the reciprocal theorem of elastodynamics as

$$
c_{\alpha \beta}(\boldsymbol{\xi}) u_{\beta}(\boldsymbol{\xi}, \omega)=\int_{\Gamma}\left[u_{\alpha \beta}^{*}(\boldsymbol{\xi}, \mathbf{x}, \omega) t_{\beta}(\mathbf{x}, \omega)-t_{\alpha \beta}^{*}(\boldsymbol{\xi}, \mathbf{x}, \omega) u_{\beta}(\mathbf{x}, \omega)\right] \mathrm{d} \Gamma(\mathbf{x}), \quad \alpha, \beta=1,2,
$$

where $u_{\alpha \beta}^{*}$ and $t_{\alpha \beta}^{*}$ are the full-space frequency domain elastodynamic fundamental solution displacement and traction tensors, respectively [18]. Note that $u_{\alpha \beta}^{*}(\boldsymbol{\xi}, \mathbf{x}, \omega)$ and $t_{\alpha \beta}^{*}(\boldsymbol{\xi}, \mathbf{x}, \omega)$ represent the $\beta$ component of the displacement and the traction on the boundary, respectively, at the point $\mathbf{x}$ due to a unit time-harmonic load of angular frequency $\omega$ applied at the point $\xi$ in the direction $\alpha$. Also, $u_{\beta}, t_{\beta}$ are frequency domain displacements and tractions on the boundary, $\omega$ stands for the angular frequency and $c_{\alpha \beta}$ is called the jump coefficient given by

$$
c_{\alpha \beta}(\xi)= \begin{cases}\frac{1}{2} \delta_{\alpha \beta} & \text { if } \Gamma \text { is smooth at point } \xi \\ c_{\alpha \beta} & \text { if } \Gamma \text { has a corner at point } \xi\end{cases}
$$

where $\delta_{\alpha \beta}$ represents the Kronecker delta. The jump coefficient for corner points can be derived by an indirect approach as described in [18]. The integrals in Eq. (2) are interpreted in the sense of the Cauchy principal value.

Let us denote as $\Gamma_{1}$ the part of the boundary corresponding to the crack face, where tractions are applied, and $\Gamma_{2}$ the remaining part of the fictitious vertical boundary of the quarter-space that is discretized. Let $\Gamma_{0}$ be the part of the traction-free surface of the quarter-space which will be included in the discretization and $\Gamma_{\infty}$ the remaining infinite part which will be omitted (see Fig. 4). In this case, Eq. (2) becomes

$$
\begin{aligned}
& c_{\alpha \beta}(\boldsymbol{\xi}) u_{\beta}(\boldsymbol{\xi}, \omega)+\int_{\Gamma_{\infty}} t_{\alpha \beta}^{*}(\boldsymbol{\xi}, \mathbf{x}, \omega) u_{\beta}(\mathbf{x}, \omega) \mathrm{d} \Gamma(\mathbf{x})+\int_{\Gamma_{0} \cup \Gamma_{1} \cup \Gamma_{2}} t_{\alpha \beta}^{*}(\boldsymbol{\xi}, \mathbf{x}, \omega) u_{\beta}(\mathbf{x}, \omega) \mathrm{d} \Gamma(\mathbf{x}) \\
& \quad=\int_{\Gamma_{1} \cup \Gamma_{2}} u_{\alpha \beta}^{*}(\boldsymbol{\xi}, \mathbf{x}, \omega) t_{\beta}(\mathbf{x}, \omega) \mathrm{d} \Gamma(\mathbf{x}) .
\end{aligned}
$$



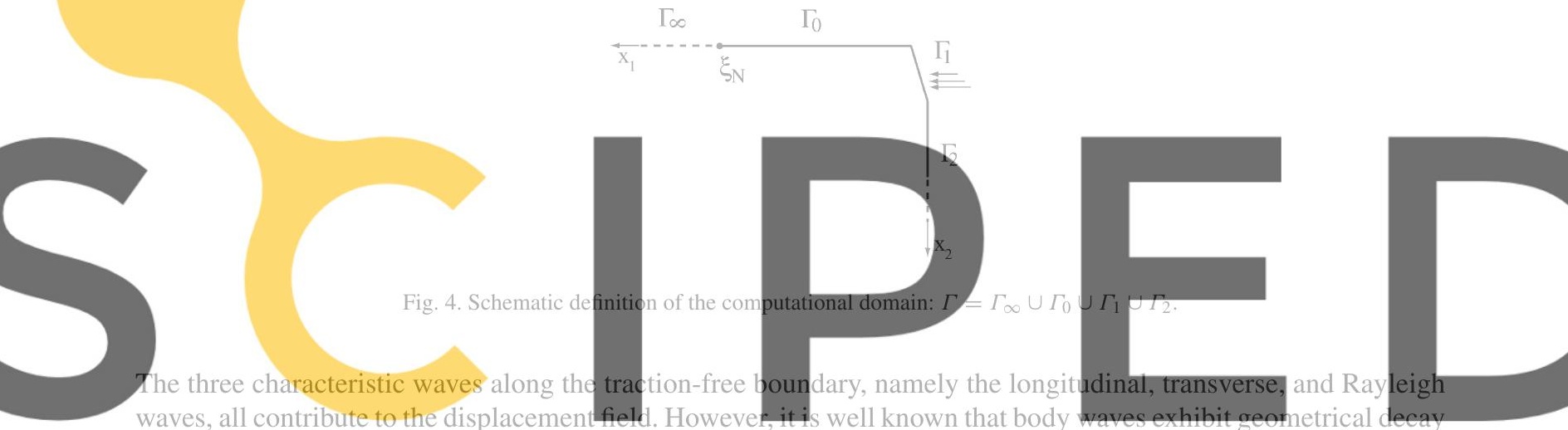

in the propagating direction, whereas Rayleigh waves in two dimensions do not. Body waves generated by a line source in an infinite medium present cylindrical wavefronts and, consequently, the amplitude of the disturbance

wave disturbances decays as $r$. By contrast, Rayleigh waves produced by a line source in a half-space present plane wavefronts and the amplitude of the corresponding disturbance does not decay with $r$. Therefore, the displacement far-field solution can be approximated by the Rayleigh surface wave component of the solution, thereby neglecting the contribution of the body waves. Hence, if the truncation points $\boldsymbol{\xi}_{N}$ is located far enough from the source region, then we can write for the infinite boundary $\Gamma_{\infty}$

$$
\boldsymbol{\xi} \in \Gamma_{\infty}: u_{\alpha}(\boldsymbol{\xi}, \omega) \approx R(\omega) u_{\alpha}^{\mathrm{SR}}(\boldsymbol{\xi}, \omega),
$$

where $R$ is the unknown complex amplitude of the far-field Rayleigh wave and $u_{\alpha}^{\mathrm{SR}}$ represents the frequency domain displacements corresponding to a unit-amplitude time-harmonic Rayleigh surface wave of angular frequency $\omega$ propagating along the surface of the quarter-space in the positive direction. The expressions for $u_{\alpha}^{\mathrm{SR}}$ can be found, for instance, in [19].

Hence, Eq. (2) can be rewritten as

$$
\begin{aligned}
& c_{\alpha \beta}(\boldsymbol{\xi}) u_{\beta}(\boldsymbol{\xi}, \omega)+R(\omega) I_{\alpha}(\boldsymbol{\xi}, \omega)+\int_{\Gamma_{0} \cup \Gamma_{1} \cup \Gamma_{2}} t_{\alpha \beta}^{*}(\boldsymbol{\xi}, \mathbf{x}, \omega) u_{\beta}(\mathbf{x}, \omega) \mathrm{d} \Gamma(\mathbf{x}) \\
& \quad=\int_{\Gamma_{1} \cup \Gamma_{2}} u_{\alpha \beta}^{*}(\boldsymbol{\xi}, \mathbf{x}, \omega) t_{\beta}(\mathbf{x}, \omega) \mathrm{d} \Gamma(\mathbf{x}),
\end{aligned}
$$

where

$$
I_{\alpha}(\boldsymbol{\xi}, \omega):=\int_{\Gamma_{\infty}} t_{\alpha \beta}^{*}(\boldsymbol{\xi}, \mathbf{x}, \omega) u_{\beta}^{\mathrm{SR}}(\mathbf{x}, \omega) \mathrm{d} \Gamma(\mathbf{x})
$$

Note that in Eq. (6) the complex amplitude $R$ is unknown, but the integrand in Eq. (7) is known. Therefore, the integrals $I_{\alpha}(\xi, \omega)$ may be approximated numerically. Here, however, we propose a more elegant approach based on the reciprocity theorem of elastodynamics by which the integral $I_{\alpha}(\xi, \omega)$ is replaced by an integral over a finite domain. Let us consider a harmonic Rayleigh surface wave of frequency $\omega$ and unit-amplitude propagating along the free surface of the corresponding half-space in the positive direction. For the computation of $I_{\alpha}(\xi, \omega)$ we introduce a vertical fictitious boundary of infinite extent. Let us choose the Rayleigh surface wave as one elastodynamic state and the time-harmonic full-space fundamental solution of the same frequency $\omega$ as the other elastodynamic state. By virtue of the reciprocity theorem of elastodynamics, and after the limiting process of taking the observation point $\xi$ to the boundary, an integral representation may be derived for the quarter-space as

$$
c_{\alpha \beta}(\boldsymbol{\xi}) u_{\beta}^{\mathrm{SR}}(\boldsymbol{\xi}, \omega)=\int_{\Gamma}\left[u_{\alpha \beta}^{*}(\boldsymbol{\xi}, \mathbf{x}, \omega) t_{\beta}^{\mathrm{SR}}(\mathbf{x}, \omega)-t_{\alpha \beta}^{*}(\boldsymbol{\xi}, \mathbf{x}, \omega) u_{\beta}^{\mathrm{SR}}(\mathbf{x}, \omega)\right] \mathrm{d} \Gamma(\mathbf{x}) .
$$


Invoking the zero traction boundary conditions along $\Gamma_{0}$ and $\Gamma_{\infty}$, Eq. (8) becomes

$\int_{\Gamma_{\infty}} t_{\alpha \beta}^{*}(\xi, \mathbf{x}, \omega) u_{\beta}^{\mathrm{SR}}(\mathbf{x}, \omega) \mathrm{d} \Gamma(\mathbf{x})=-c_{\alpha \beta}(\xi) u_{\beta}^{\mathrm{SR}}(\xi, \omega)-\int_{\Gamma_{0} \cup \Gamma_{1} \cup \Gamma_{2}} t_{\alpha \beta}^{*}(\xi, \mathbf{x}, \omega) u_{\beta}^{\mathrm{SR}}(\mathbf{x}, \omega) \mathrm{d} \Gamma(\mathbf{x})$
$\longrightarrow$
Note that along the fictitious vertical boundary the
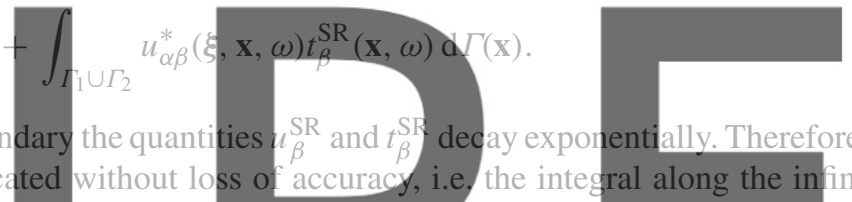
re, the infinite
boundary can be extended to $\Gamma_{1} \cup \Gamma_{2}$ only. Ed. (9) allows us to compute the integrals $I_{\alpha}(\xi, \omega)$, in terms of integrals
over the bounded boundaries of the problem. This step constitutes the key to the present approach and provides a
term in Eq. (6).
Through Eq. (5), the solution is described asymptotically as a Rayleigh wave of unknown amplitude and phase.
In order to eliminate the unknown $R$ in Eq. (6), the solution in the computational domain is matched to the far-field giste polfior af fheed
\[ R(\omega) \approx \frac{u_{\alpha}\left(\xi_{N}, \omega\right)}{u_{\alpha}^{S R}\left(\xi_{N}, \omega\right)}, \quad \alpha=1,2 . \]

we can define then

$$
A_{\alpha}(\xi, \omega):=\frac{I_{\alpha}(\boldsymbol{\xi}, \omega)}{u_{\alpha}^{\mathrm{SR}}\left(\boldsymbol{\xi}_{N}, \omega\right)}, \quad \alpha=1,2
$$

With these definitions, Eq. (6) can be rewritten as

$$
\begin{gathered}
c_{\alpha \beta}(\boldsymbol{\xi}) u_{\beta}(\boldsymbol{\xi}, \omega)+A_{\alpha}(\boldsymbol{\xi}, \omega) u_{\alpha}\left(\boldsymbol{\xi}_{N}, \omega\right)+\int_{\Gamma_{0} \cup \Gamma_{1} \cup \Gamma_{2}} t_{\alpha \beta}^{*}(\boldsymbol{\xi}, \mathbf{x}, \omega) u_{\beta}(\mathbf{x}, \omega) \mathrm{d} \Gamma(\mathbf{x}) \\
=\int_{\Gamma_{1} \cup \Gamma_{2}} u_{\alpha \beta}^{*}(\boldsymbol{\xi}, \mathbf{x}, \omega) t_{\beta}(\mathbf{x}, \omega) \mathrm{d} \Gamma(\mathbf{x}), \quad \alpha=1,2 .
\end{gathered}
$$

It is convenient to cast Eq. (12) in matrix form as follows:

$$
\mathbf{c}(\boldsymbol{\xi}) \mathbf{u}(\boldsymbol{\xi}, \omega)+\mathbf{A}(\boldsymbol{\xi}, \omega) \mathbf{u}\left(\xi_{N}, \omega\right)+\int_{\Gamma_{0} \cup \Gamma_{1} \cup \Gamma_{2}} \mathbf{t}^{*}(\boldsymbol{\xi}, \mathbf{x}, \omega) \mathbf{u}(\mathbf{x}, \omega) \mathrm{d} \Gamma(\mathbf{x})=\int_{\Gamma_{1} \cup \Gamma_{2}} \mathbf{u}^{*}(\boldsymbol{\xi}, \mathbf{x}, \omega) \mathbf{t}(\mathbf{x}, \omega) \mathrm{d} \Gamma(\mathbf{x}),
$$

where

$$
\mathbf{A}(\boldsymbol{\xi}, \omega)=\left[\begin{array}{cc}
A_{1}(\boldsymbol{\xi}, \omega) & 0 \\
0 & A_{2}(\boldsymbol{\xi}, \omega)
\end{array}\right] .
$$

It should be noted that Eq. (13) corresponds to the standard terms of the simply truncated boundary integral equation to which a correction term $\mathbf{A}(\boldsymbol{\xi}, \omega) \mathbf{u}\left(\boldsymbol{\xi}_{N}, \omega\right)$ has been added to account for the contribution of the omitted part of the boundary of the quarter-space on which a Rayleigh surface wave is assumed to be predominant.

According to Eq. (13), the computational boundary that needs to be discretized is $\Gamma_{0} \cup \Gamma_{1} \cup \Gamma_{2}$. The BEM system of equations is formed in the usual manner. Following the notation in [18], the corrected BEM system may be written as

$$
\mathbf{H}(\omega) \mathbf{U}(\omega)+\underbrace{\left[\begin{array}{c}
\mathbf{A}_{1}(\omega) \\
\vdots \\
\mathbf{A}_{N}(\omega)
\end{array}\right]}_{\mathbf{A}(\omega)}\left[\begin{array}{c}
u_{1}\left(\boldsymbol{\xi}_{N}, \omega\right) \\
u_{2}\left(\boldsymbol{\xi}_{N}, \omega\right)
\end{array}\right]=\mathbf{G}(\omega) \mathbf{T}(\omega),
$$


where $\mathbf{H}$ and $\mathbf{G}$ are the BEM displacement and tractions system matrices, respectively, $\mathbf{U}$ and $\mathbf{T}$ are the vectors of nodal displacements and tractions, respectively, and $\mathbf{A}_{j}(\omega)=\mathbf{A}\left(\xi_{j}, \omega\right)$. From Eq. (15) it follows that the BEM displacement matrix for the corrected BEM scheme is obtained from the BEM displacement matrix $\mathbf{H}$ for the truncated system by adequately adding the correction coefficients $\mathbf{A}_{j}$ to the columns corresponding to the end node $\xi_{\text {N }}$
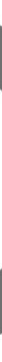

Hence, the corrected BEM system may be written as

\section{gister for (fireé(w)}

Eq. (17) constitutes the BEM system modified to account for the contribution of the far-field Rayleigh surface wave through the correction matrix $\tilde{\mathbf{A}}(\omega)$. It is noted that, by interpolating the known displacements and tractions of the Rayleigh wave far-field solution with the same element shape functions implemented in the BEM scheme, the coefficients of the correction matrix can be computed with the same element matrices used to compute the original $\mathbf{H}$ and $\mathbf{G}$ matrices. This becomes clear by comparing Eqs. (6) and (9). Indeed, if $\mathbf{u}^{\mathrm{SR}}$ and $\mathbf{t}^{\mathrm{SR}}$ are interpolated with the same element shape functions as $\mathbf{u}$ and $\mathbf{t}$, then the resulting element integrals are the same in both cases. In fact, it is easy to see that

$$
\mathbf{A}(\omega)=-\mathbf{H}(\omega) \mathbf{U}^{\mathrm{SR}}(\omega)+\mathbf{G}(\omega) \mathbf{T}^{\mathrm{SR}}(\omega)
$$

where $\mathbf{U}^{\mathrm{SR}}$ and $\mathbf{T}^{\mathrm{SR}}$ are the vectors of nodal displacements and tractions due to the unit-amplitude time-harmonic Rayleigh wave, respectively.

Consequently, no additional integrals need to be computed and the correction to account for the part of the boundary excluded from the discretization can be implemented at essentially no additional cost. It should be pointed out as well that the above presented implementation is very simple and requires minimum modification of the routine which generates the $\mathbf{H}$ and $\mathbf{G}$ matrices. Once the original matrices are computed in the usual manner, they are used to obtain the correction matrices $\mathbf{A}$ according to Eq. (18). Then, the modified matrices are generated as indicated in Eq. (17).

As a validation test for the proposed numerical scheme, a free Rayleigh pulse travelling along a two-dimensional homogeneous, isotropic, linearly elastic half-space is analyzed. This wave can be reproduced in the numerical simulation by considering a quarter-space, and by imposing on the fictitious vertical boundary the known displacements corresponding to the Rayleigh wave. Both the simply truncated and the corrected numerical schemes are employed to obtain displacements on the free surface, which are then compared to those of the Rayleigh wave. The time domain analytical displacements are obtained by convolution with the pulse. Since the main assumption exactly holds, very accurate results are obtained with the corrected numerical scheme irrespective of the location of the truncation point. For observation points far from the truncation point, both numerical solutions show very good agreement with the analytical solution. However, the truncated solution exhibits a spurious reflection from the end of the computational domain which is absent in the corrected solution (see left plot in Fig. 5). As the observation point is brought closer to the truncation point, the spurious reflection starts interfering with the direct signal which results in a clear deterioration of the accuracy of the truncated solution. On the contrary, the corrected solution retains its accuracy everywhere in the computational domain, even when the observation point coincides with the truncation point (see right plot in Fig. 5). In addition, as expected, the numerically obtained values for the complex amplitude of the far-field Rayleigh wave 


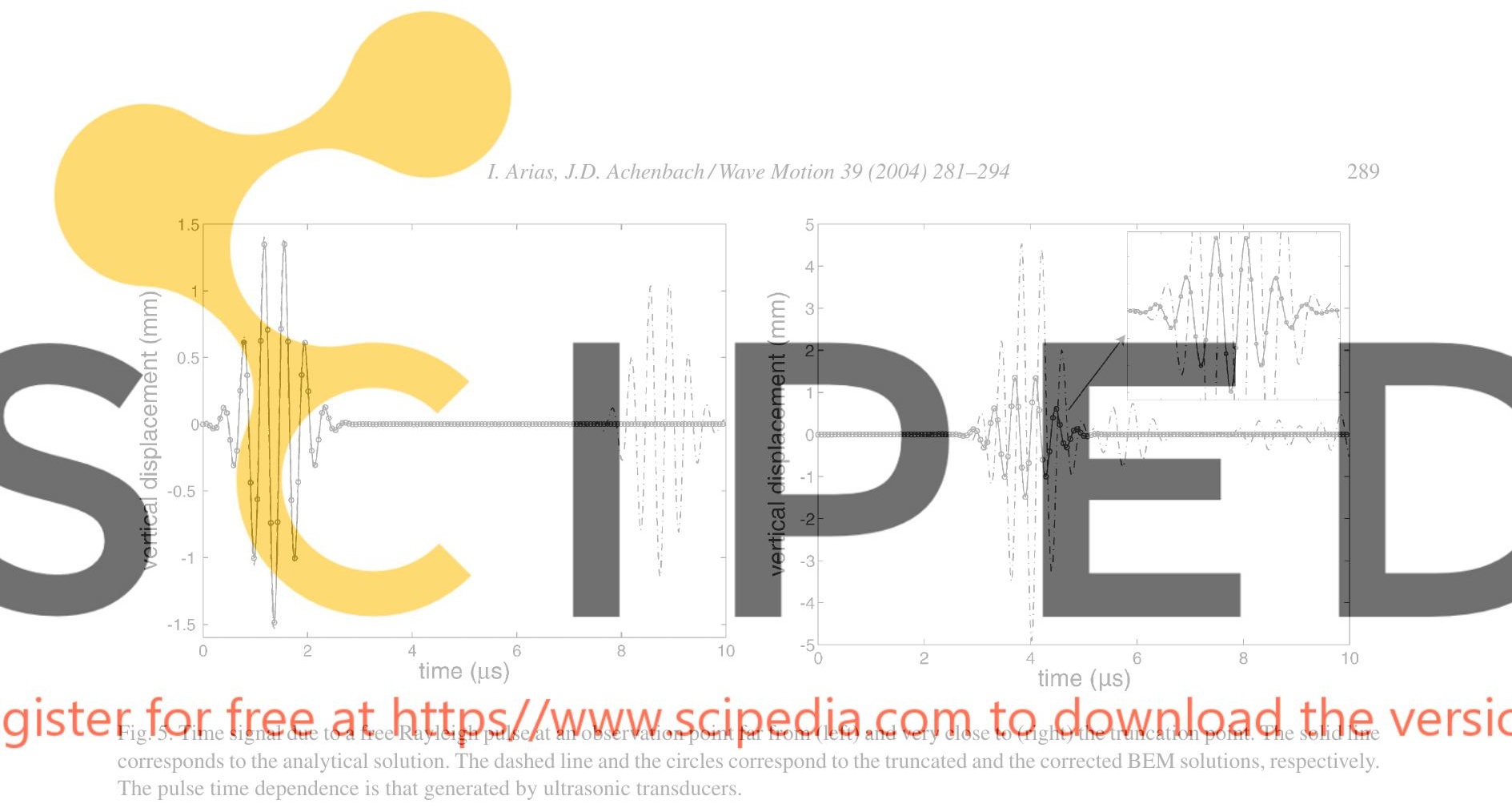

in the frequency domain $R\left(\omega_{i}\right)$, for all considered frequencies $\omega_{i}$, coincide with the discrete Fourier transform of the pulse.

\section{Numerical simulations}

We illustrate the computational method through selected numerical examples of nucleating and propagating, surface-breaking and buried cracks in an aluminum half-space. The nucleation and propagation of cracks in a two-dimensional geometry generates both attenuating body waves and non-attenuating Rayleigh surface waves. Therefore, the accuracy of the computed disturbances at the surface strongly depends on the adequate selection of the truncation point. From the previous sections, we can anticipate that the corrected solution should be accurate for all times and everywhere in the computational domain, as long as the truncation point is chosen far enough for the body waves to be negligible at this point. If the truncation point is located too close to the source region, the present method provides an inaccurate solution. Unlike in the case of the simple truncation method, in which the error of the solution can be identified in general as a spurious reflection, the inaccurate corrected solution is difficult to interpret in physical terms. From a practical point of view, the distance at which the body waves have sufficiently attenuated to become negligible can be estimated from the frequency content of the signal. In principle, the dominant frequencies guide the selection of the truncation point (for superficial loads, for instance, the relative significance of the body waves components with respect to the Rayleigh wave component decreases with increasing frequency), as well as the elements size (the general rule is to have 10 nodes per wavelength). If the frequency spectrum of the signal is relatively narrow, a representative frequency can be identified and the design of the mesh (truncation point and element size) can be based on this frequency. If the frequency spectrum is broad, different meshes tailored to each frequency can be used. Here, the truncation point on the surface is located at a distance of 60 Rayleigh wavelengths from the symmetry axis. Note that the fictitious boundary along the symmetry axis does not carry Rayleigh surface waves. Thus, the simple truncation of this boundary at a sufficient distance leads to accurate results and no correction is needed. Here, this boundary is truncated at a distance of 70 Rayleigh wavelengths from the surface.

As a first example, we study the acoustic emission from a very small nucleating surface-breaking crack. In the limit, the nucleation of a small surface-breaking crack near the surface can be modeled as a surface dipole. As noted in [7], this representation is qualitatively similar to the shear traction dipole model for laser generation of ultrasound. 

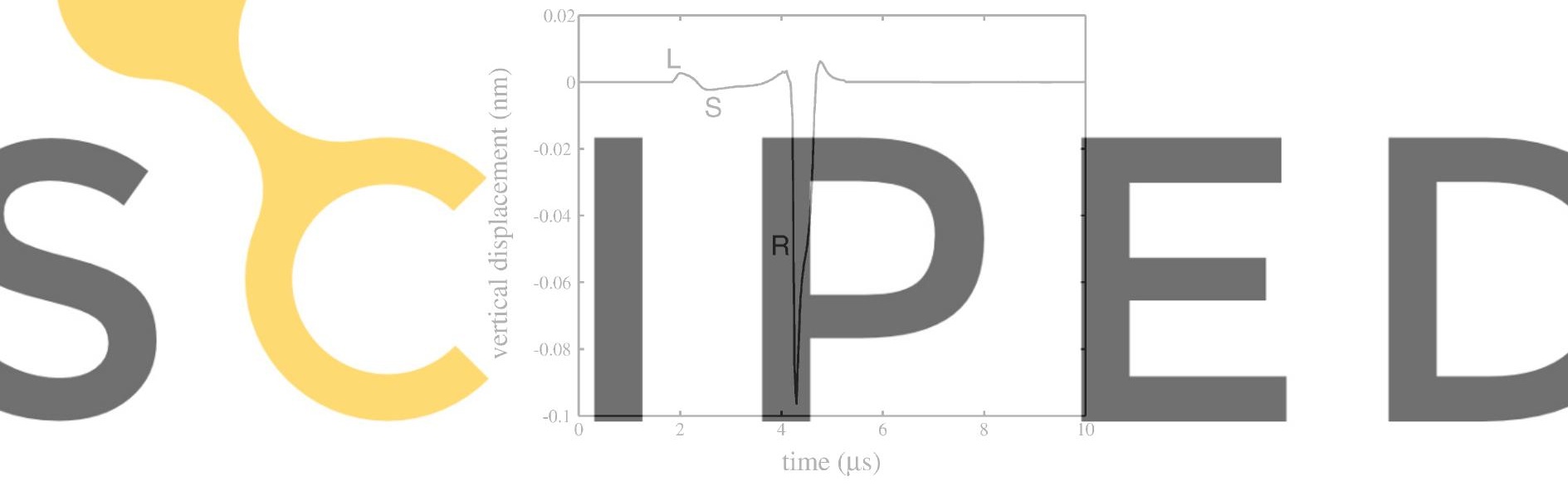

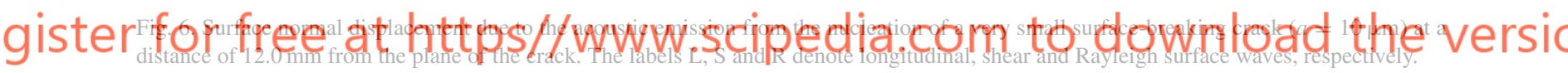

Fig. 6 shows that indeed, for a small, nucleating surface-breaking crack, the generated far-field waveform tends to the characteristic monopolar Rayleigh wave-dominated signal generated by a concentrated line-focused laser source with impulsive time dependence for the pulse [20].

The asymptotic analysis presented in [7] predicts that the peak amplitude $A$ of the acoustic emission pulse is proportional to the rate of change of size of the defect, i.e. $A \propto \Delta a / \Delta t$. Fig. 7 shows the waveforms corresponding to the nucleation of three surface-breaking cracks of different sizes, for a fixed $S_{\alpha}(t)$, i.e. for a fixed $\Delta t$. For increasing $\Delta a$, we observe in the waveforms the corresponding increases in the peak amplitude, consistent with the predictions of Scruby [7]. Note that the reported surface disturbances are recorded far enough from the defect to be considered far-field. Closer to the nucleating crack, these trends are not so clear. The increasing length of the nucleating crack also reflects in the extent of the time signal.

The same trend is observed in the propagation of a surface-breaking crack. Three different growths $\Delta a$ have been considered for a fixed $S_{\alpha}(t)$, i.e. for a fixed $\Delta t$, and the corresponding waveforms at the surface are depicted in Fig. 8 for distances of 3 and $16 \mathrm{~mm}$ from the plane containing the crack.

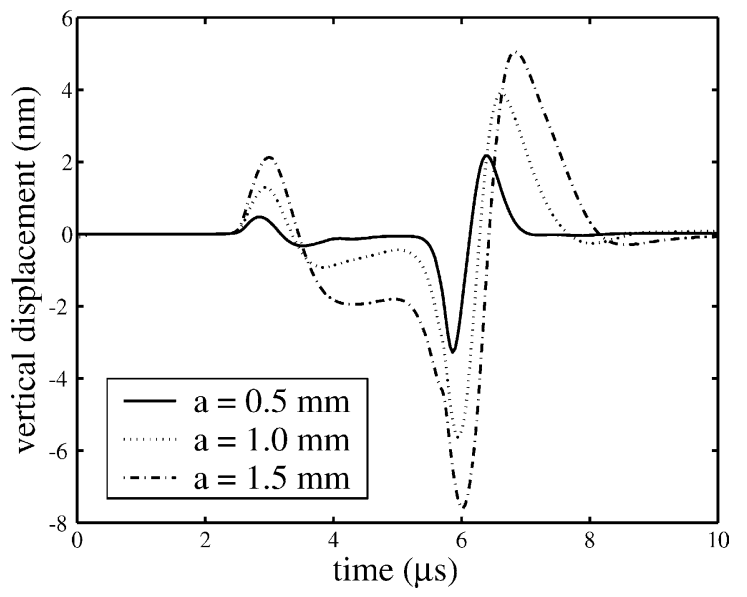

Fig. 7. Surface normal displacement due to the acoustic emission from the nucleation of surface-breaking cracks of different lengths $a$ at $16.0 \mathrm{~mm}$ distance from the plane of the crack. 

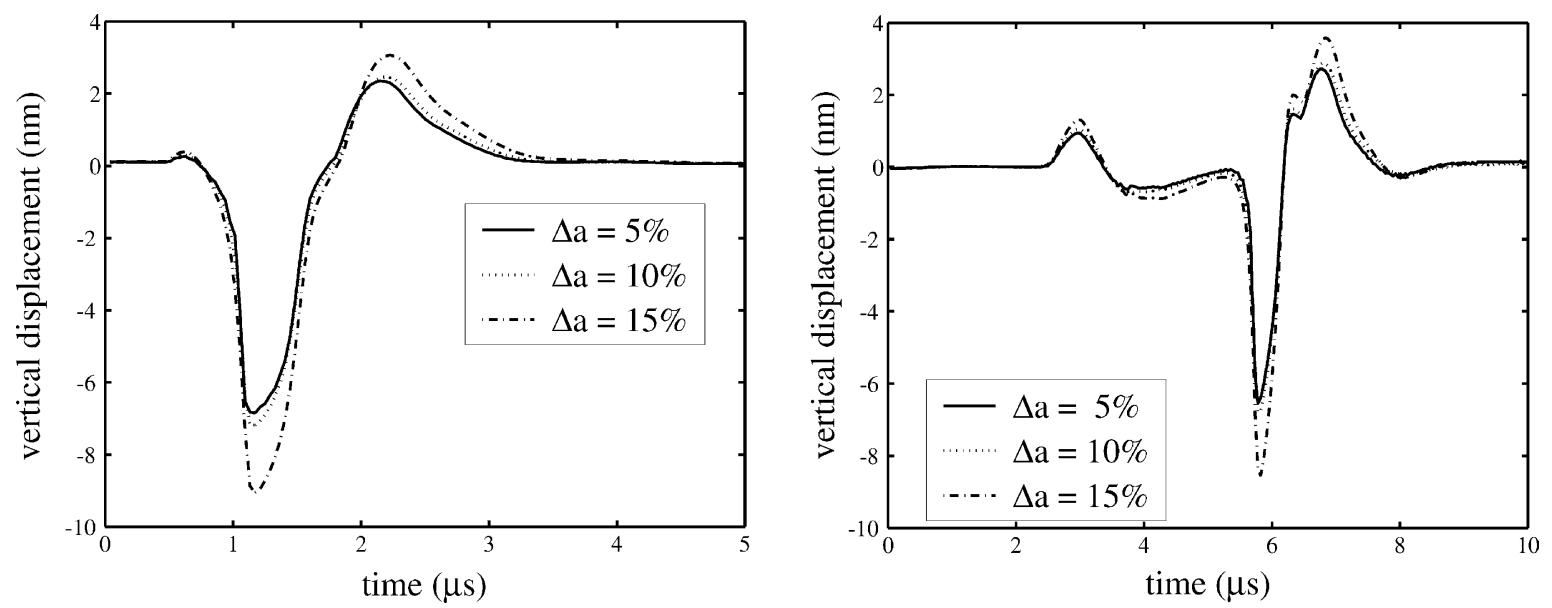

Fig. 8. Surface normal displacement due to the acoustic emission from the propagation of a surface-breaking crack $(a=1.0 \mathrm{~mm})$ for different growth lengths $\Delta a$, at distances of $3 \mathrm{~mm}$ (left) and $16 \mathrm{~mm}$ (right) from the plane of the crack.

The acoustic emissions from the nucleation and propagation of buried cracks are studied next. Fig. 9 shows the normal surface disturbances originated from the nucleation of three surface-breaking cracks of different sizes, for a given depth defined by the midpoint of the crack. Similar to the case of surface-breaking cracks, the peak amplitude increases with the crack size for a given stress release rate. Fig. 10 presents surface normal displacements from the propagation of a crack of $1.0 \mathrm{~mm}$ length buried a distance of $1.0 \mathrm{~mm}$ from the surface to the mid point of the crack. The crack is assumed to propagate upwards since the crack tip closer to the surface exhibits a larger SIF [16]. Three different crack growth extents are considered and again the peak amplitude increases with increasing crack growth length for a fixed $\Delta t$. Qualitatively, the waveforms for a nucleating and a propagating buried crack are quite similar. It is apparent that in the waveforms shown in Fig. 10 the Rayleigh wave component is more significant due to the fact that the cracks in this case are closer to the surface. The effect of the depth of the crack on the surface displacement waveforms has been studied by considering the nucleation of crack of a specific size located at different distances

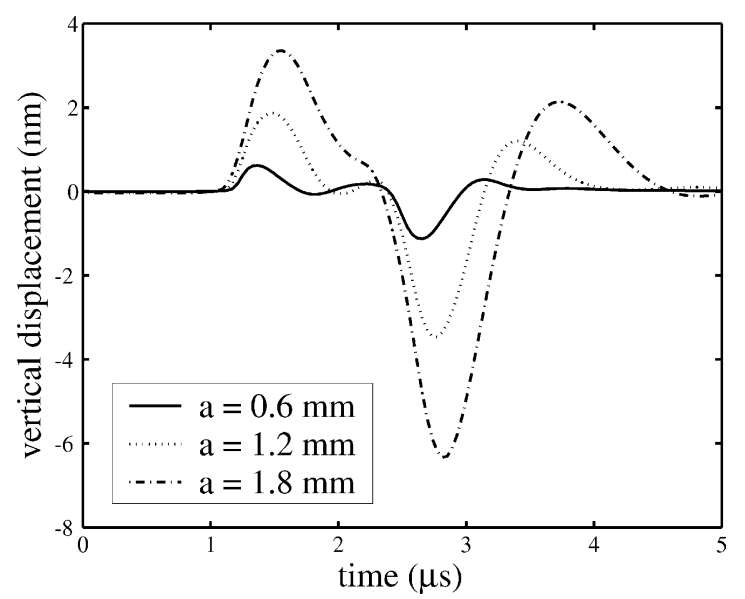

Fig. 9. Surface normal displacement due to the acoustic emission from the nucleation of buried cracks of different lengths $a$ at a distance of $5.0 \mathrm{~mm}$ from the plane of the crack. The midpoints of the cracks are located at a depth $d=5.0 \mathrm{~mm}$ beneath the surface. 

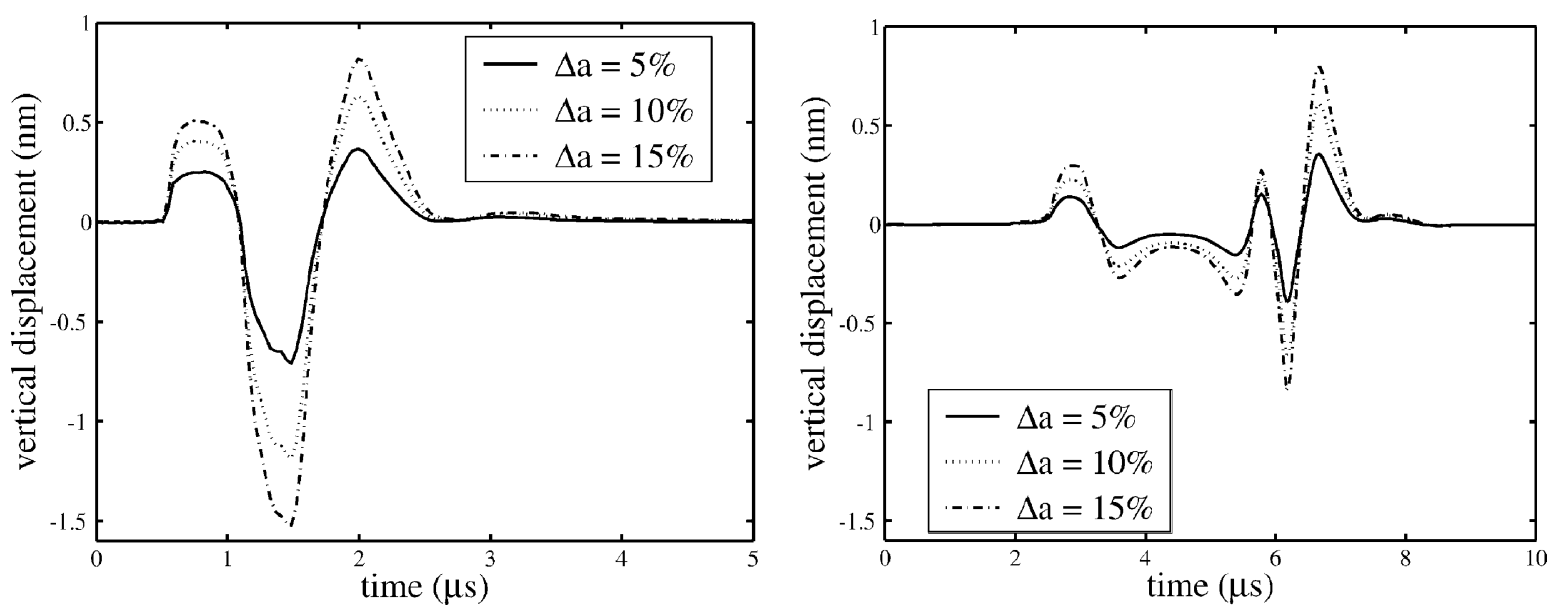

Fig. 10. Surface normal displacement due to the acoustic emission from the propagation of a buried crack $(a=1.0 \mathrm{~mm}$ and $d=0.5 \mathrm{~mm})$ for different growth lengths $\Delta a$, at distances of $4 \mathrm{~mm}$ (left) and $16 \mathrm{~mm}$ (right) from the plane of the crack.

beneath the surface. The corresponding surface disturbances at a distance of $5.0 \mathrm{~mm}$ from the plane of the crack are shown in Fig. 11. As pointed out, the relative significance of the Rayleigh wave component with respect to the body wave components decreases as the crack depth increases.

In addition, it can be noted in Fig. 11 that the amplitude of the signals exhibit a maximum for an intermediate depth which suggests an angular dependency in the amplitude of acoustic emission signals. This has been further investigated by considering the variation along the surface of the half-space of the acoustic emission from a specific buried crack. Fig. 12 displays the normal surface displacements at different observation points due to the nucleation of a crack of length $a=1.0 \mathrm{~mm}$ located at a distance of $d=1.0 \mathrm{~mm}$ beneath the surface. It can be noted that as the observation point departs from the crack position, the amplitude increases, reaches a maximum, and then decreases in the far-field. This observation is consistent with the results on the angular variation of the amplitude of the acoustic emission reported in [8].

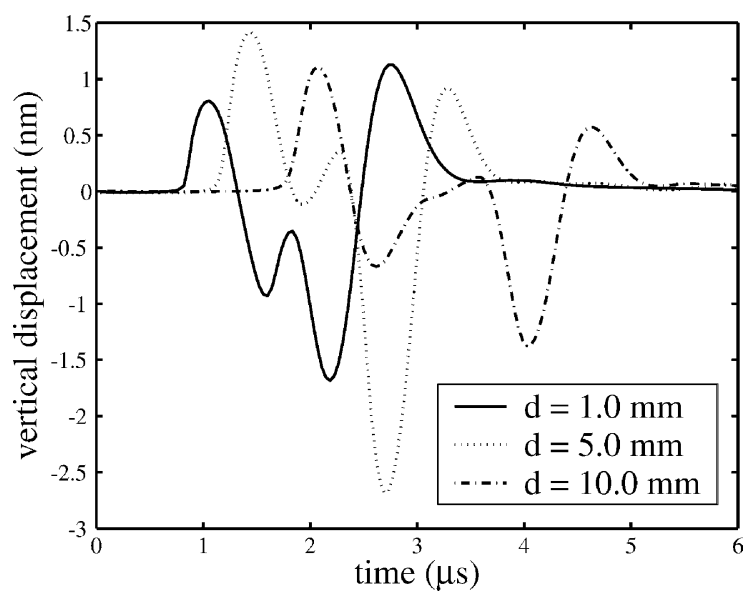

Fig. 11. Surface normal displacement due to the acoustic emission from the nucleation of buried cracks of length $a=1.0 \mathrm{~mm}$ at a distance of $5.0 \mathrm{~mm}$ from the plane of the crack. The midpoints of the cracks are located at different depths $d$ beneath the surface. 

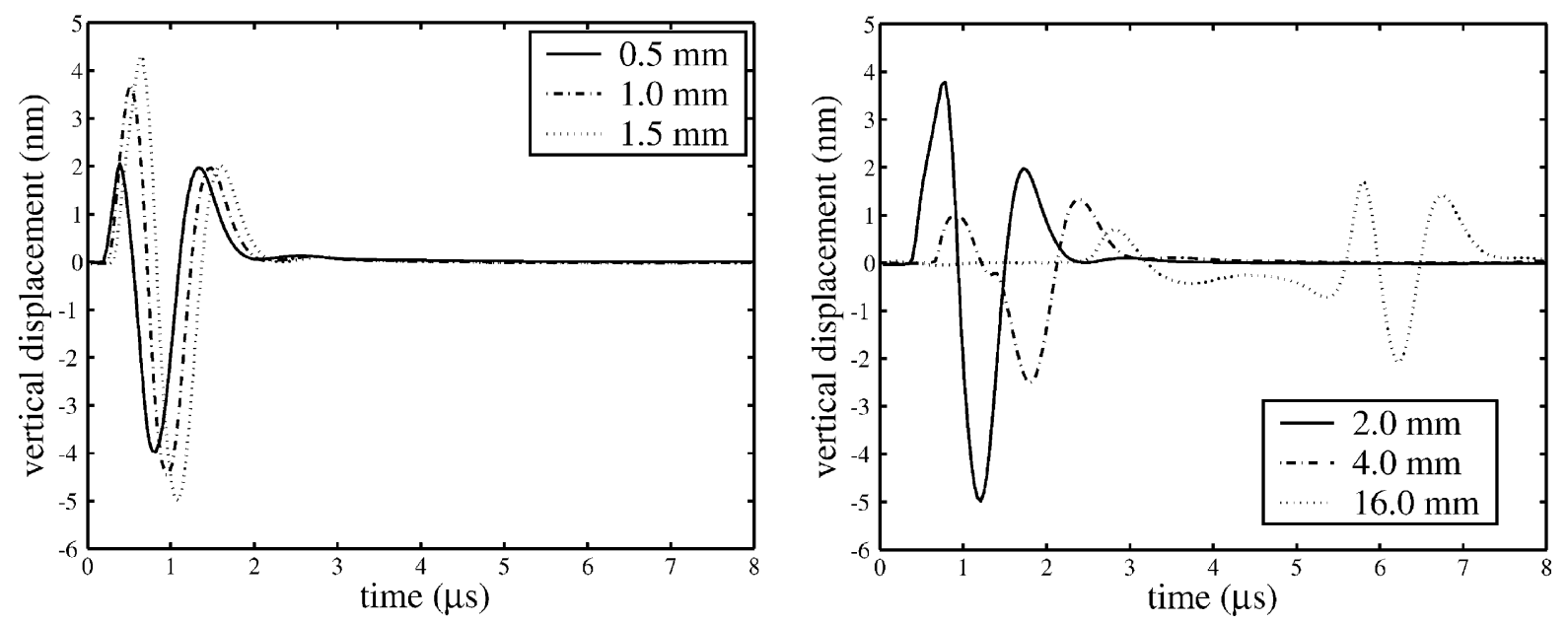

Fig. 12. Surface normal displacement due to the acoustic emission from the nucleation of a buried crack $(a=1.0 \mathrm{~mm}$ and $d=1.0 \mathrm{~mm})$ at different distances from the plane of the crack.

\section{Conclusions}

The acoustic emissions from the nucleation and propagation of surface-breaking and buried cracks have been calculated. A computational approach based on the boundary element method has been implemented. By the use of reciprocity considerations, a correction method for the truncation of the infinite boundary has been presented, which allows the undamped Rayleigh waves to escape the computational domain without producing spurious reflections from its limits. This technique produces numerical solutions which are highly accurate everywhere in the computational domain and for all computed times. This is particularly useful for studies of acoustic emission, since the observation point, which is often located far from the source region, can be brought close to the truncation point without loss of accuracy.

It has been shown that in the limit of a small nucleating surface-breaking crack, the surface disturbances tend to those generated by a shear dipole at the surface, as has been pointed out in previous studies. We have analyzed the effect of the size of the nucleating crack and the length of the crack growth for both surface-breaking and buried cracks. In addition, we have studied the effect of the buried depth in the surface disturbances originated form nucleating buried cracks. The analysis of the acoustic emission signals for nucleating buried cracks at different observation locations along the surface of the half-space has shown evidence of an angular dependence consistent with previous theoretical studies.

\section{Acknowledgements}

This paper is based upon the work partially supported by the Federal Aviation Administration under contract No. DFTA 03-98-F-IA029, and partially supported by the Office of Naval Research under contract No. N00014-89-J-1362.

\section{References}

[1] J. Watson, Advanced implementation of the boundary element method for two- and three-dimensional elastostatics, in: P. Banerjee, R. Butterfield (Eds.), Developments in Boundary Element Methods, I, Elsevier, London, 1979, pp. 31-63. 
[2] S. Kobayashi, N. Nishimura, Analysis of dynamic soil-structure interactions by boundary integral equation method, in: P. Lascaux (Ed.), Proceedings of the Third International Symposium on Numerical Methods Engineering, Paris, 1983, pp. 353-362.

[3] S. Bu, Infinite boundary elements for the dynamic analysis of machine foundations, Int. J. Numer. Meth. Eng. 40 (21) (1997) $3901-3917$.

[4] I. Arias, J. Achenbach, Rayleigh wave correction for the BEM analysis of two-dimensional elastodynamic problems in a half-space, Int. J. Numer. Meth. Eng., in press.

[5] W. Marketz, F. Fischer, H. Clemens, Deformation mechanisms in TiAl intermetallics—experiments and modeling, Int. J. Plasticity 19 (2003) 281-321.

[6] P. Mummery, B. Derby, C. Scruby, Acoustic emission from particulate-reinforced metal matrix composites, Acta Metall. Mater. 41 (5) (1993) 1431-1445.

[7] C. Scruby, Quantitative acoustic emission techniques, in: Research Techniques in Nondestructive Testing, vol. VIII, Academic Press, London, 1985, pp. 141-210.

[8] J. Achenbach, J. Harris, Acoustic emission from a brief crack propagation event, ASME J. Appl. Mech. 46 (1979) $107-112$.

[9] Z. Gong, D. DuQuesnay, S. McBride, Measurement and interpretation of fatigue crack growth in 7075 aluminum alloy using acoustic emission monitoring, J. Testing Eval. 26 (6) (1998) 567-574.

[10] R. Pensec, A. Laksimi, C. Herve, M. Cherfaoui, Discrimination of flaws evolution patterns during pressure vessels static and dynamic cycles of loading thanks to the acoustic emission method, in: D. Thompson, D. Chimenti (Eds.), Review of Progress in Quantitative Nondestructive Evaluation, vol. 19, AIP Press, New York, 2000, pp. 375-382.

[11] Z. Shi, J. Jarzynski, S. Bair, S. Hurlebaus, L. Jacobs, Characterization of acoustic emission signals from fatigue fracture, J. Mech. Eng. Sci. 214 (9) (2000) 1141-1149.

[12] J. Choi, J. Luo, I. Daniel, Analysis of acoustic emission waveforms from propagating fatigue crack, in: D. Thompson, D. Chimenti (Eds.), Review of Progress in Quantitative Nondestructive Evaluation, vol. 19, AIP Press, New York, 2000, pp. 351-358.

[13] C. Scruby, R. Dewhurst, D. Hutchins, S. Palmer, Quantitative studies of thermally-generated elastic waves in laser irradiated metals, J. Appl. Phys. 51 (1980) 6210-6216.

[14] L. Rose, The stress-wave radiation from growing cracks, Int. J. Fract. 17 (1981) 45-60.

[15] J. Harris, J. Pott, Surface motion excited by acoustic emission from a buried crack, ASME J. Appl. Mech. 51 (1984) 77-83.

[16] H. Tada, P. Paris, G. Irwin, The Stress Analysis of Cracks Handbook, 3rd ed., The American Society of Mechanical Engineers, 2000.

[17] G. Blandford, A. Ingraffea, J. Liggett, Two-dimensional stress intensity factor computations using the boundary element method, Int. J. Numer. Meth. Eng. 17 (1981) 387-404.

[18] J. Domínguez, Boundary Elements in Dynamics, Computational Mechanics Publications, Elsevier, Southampton, UK, 1993.

[19] J. Achenbach, Wave Propagation in Elastic Solids, North-Holland/Elsevier, Amsterdam, 1973.

[20] I. Arias, J. Achenbach, Thermoelastic generation of ultrasound by line-focused laser irradiation, Int. J. Solids Struct. 40 (2004) $6917-6935$. 